\title{
O impacto das experiências emocionais positivas precoces e das competências autocompassivas na sintomatologia depressiva: 0 papel mediador de diferentes processos de regulação emocional
}

\section{The impact of early emotional positive experiences and self-compassionate abilities on depressive symptomatology: The mediator role of different emotional regulation processes}

\author{
Maria João Varela (1) \\ Cláudia Ferreira (1) \\ (1) Center for Research in Neuropsychology and Cognitive and Behavioral Intervention- University of Coimbra, Portugal \\ Recebido: 11/08/2018; Revisto: 12/12/2018; Aceite: 21/12/2018.
}

https://doi.org/10.31211/rpics.2019.5.1.101

\begin{abstract}
Resumo
Contexto: A literatura tem documentado que as experiências emocionais precoces e as competências autocompassivas desempenham um papel central na regulação emocional adaptativa e na saúde mental. Adicionalmente, crescente evidência suporta que diferentes processos de regulação emocional exercem um papel mediador significativo na relação entre experiências emocionais e sintomatologia depressiva. Métodos e Objetivo: Este estudo foi conduzido numa amostra da população geral constituída por 389 participantes, com idades entre os 18 e os 50 anos $(M=31,84 ; D P=10,97)$. Os participantes completaram um protocolo de medidas de autorresposta que avalia memórias precoces de calor e segurança, autocompaixão, proximidade e conexão aos outros, evitamento experiencial e sintomatologia depressiva. O estudo examinou um modelo integrador no qual foi colocado como hipótese que a relação entre memórias afiliativas positivas e sintomatologia depressiva é mediada pela proximidade e conexão aos outros e o evitamento experiencial. Resultados: Os resultados confirmaram a adequabilidade do modelo testado, o qual explica 34\% da variância da sintomatologia depressiva. Os resultados revelaram que experiências precoces de calor, afeto e segurança e as competências autocompassivas se associam a menores níveis de sintomatologia depressiva, através de níveis superiores de sentimentos de segurança e proximidade ao outro e menor adoção de estratégias de evitamento experiencial. Conclusões: Este estudo contribui para a clarificação do impacto das vivências emocionais precoces e das competências autocompassivas na saúde mental. Os dados sublinham a importância das competências sociais e afiliativas e de aceitação, enquanto mecanismos mediadores na explicação da saúde mental.
\end{abstract}

Palavras-Chave: Competências autocompassivas; Evitamento experiencial; Experiências precoces; Ligação ao outro; Sintomatologia depressiva.

\section{DI\&D | ISMT}

rpics@ismt.pt

https://rpics.ismt.pt
Publicação em Acesso Aberto

(02019. O(s) Autor(es). Este é um artigo de acesso aberto distribuído sob a Licença Creative Commons Attribution, que permite uso, distribuição e reprodução sem restrições em qualquer meio, desde que 0 trabalho original seja devidamente citado.

\section{Cláudia Ferreira}

CINEICC, Faculdade de Psicologia e Ciências da Educação da Universidade de Coimbra.

Rua do Colégio Novo, apartado 6153

3001-802, Coimbra, Portugal

e-mail: claudiaferreira@fpce.uc.pt 


\begin{abstract}
Background: The scientific literature supports that early emotional memories of warmth and safeness and selfcompassionate abilities play a central role in adaptive emotion regulation and mental health. Further, it is pointed out that distinct mechanisms may mediate the association between emotional experiences and depressive symptoms. Method and Aim: Participants were 389 men and women from the general population, aged between 18 and 50 years old $(M=31.84 ; S D=$ 10.97), who completed a set of self-report measures of early memories of warmth and safeness, self-compassion, social safeness, experiential avoidance, and depression symptoms. The current study examined an integrative model that hypothesized that the association between early affiliative memories and self-compassionate abilities with depressive symptomatology are mediated by social closeness and experiential avoidance. Results: Results confirmed the adequacy of the tested model, which accounted for $34 \%$ of the depressive symptoms variance. Results revealed that early emotional memories of warmth and safeness and self-compassionate abilities were associated with increased depressive symptoms, through higher feelings of safeness and closeness within the social arena and lower adoption of maladaptive experiential avoidance strategies. Conclusions: These findings contribute to the clarification of the effect of early positive emotional memories and self-compassionate abilities on mental health. Moreover, present data highlight the importance of affiliative and acceptance competences as emotion regulation mediational mechanisms to promote mental health.
\end{abstract}

Keywords: Self-compassionate abilities; Experiential avoidance; Early experiences; Social safeness; Depressive symptomatology.

\title{
Introdução
}

As experiências emocionais precoces com os pais/cuidadores e outras figuras significativas têm sido consideradas de extrema importância para a maturação do sistema límbico e na regulação da nossa resposta emocional perante o medo e face a eventos negativos de vida (Depue \& Morrone-Strupinsky, 2005; Gilbert, 2010; Schore, 2001). Na verdade, evidências empíricas e clínicas documentam que experiências precoces positivas com as figuras de vinculação, caracterizadas pela disponibilidade, afeto e segurança, se associam positivamente a indicadores de bem-estar e saúde mental (e.g., DeHart, Pelham, \& Tennen, 2006; Marta-Simões, Ferreira, \& Mendes, 2018; Mikulincer \& Shaver, 2005). Em contraste, ambientes precoces caracterizados por vivências negativas de ameaça, abuso, rejeição ou negligência parecem funcionar como fatores de risco ou vulnerabilidade para o desenvolvimento posterior de psicopatologia (e.g., Richter, Gilbert, \& McEwan, 2009).

Embora exista amplo consenso em relação ao papel central que as experiências precoces desempenham no desenvolvimento psicossocial do indivíduo, diversos autores têm enfatizado que o foco da investigação se deve centrar, não apenas na presença ou ausência de determinadas experiências objetivas, mas sim nas memórias emocionais do indivíduo acerca dessas vivências (e.g., Gilbert, Cheung, Grandfield, Campey, \& Irons, 2003). De facto, Gilbert e colaboradores (2003) têm sugerido que perante experiências precoces semelhantes, diferentes indivíduos poderão processar emocionalmente e lidar com esses eventos de forma muito distinta. De acordo com esta leitura, mais relevante que estudar o impacto do comportamento das figuras de vinculação ou das experiências precoces objetivas (e.g., as práticas e estilos parentais) será explorar as memórias emocionais precoces associadas, isto é, avaliar como cada indivíduo se recorda de sentir nestes ambientes (e.g., sentir-se amado, cuidado, protegido ou ameaçado; Gilbert et al., 2003; Richter et al., 2009).

Diversos estudos têm mostrado que a escassez de memórias emocionais precoces positivas pode assumir um papel central na explicação da psicopatologia, associando-se a maiores dificuldades na regulação das 
emoções (Gilbert, 2005, 2010; Gilbert et al., 2009). Paralelamente, a literatura tem vindo a demonstrar que a maior acessibilidade a memórias emocionais precoces positivas desempenha um efeito protetor face ao impacto de experiências/acontecimentos negativos no sofrimento e em diversos tipos de psicopatologia, nomeadamente na sintomatologia depressiva (Irons, Gilbert, Baldwin, Baccus, \& Palmer, 2006). Especificamente, estudos têm corroborado que a recordação de ambientes precoces calorosos, capazes de transmitir experiências de apoio, segurança e cuidado parecem associar-se a níveis mais elevados de bemestar e a maior resiliência face a falhas, insucessos ou fracassos, assim como a níveis mais elevados de autocompaixão (Irons et al., 2006; Kelly \& Dupasquier, 2016).

O constructo de compaixão tem sido definido na literatura em psicologia de diferentes formas. De acordo com a filosofia budista, compaixão traduz uma atitude de abertura e disponibilidade perante a dor e sofrimento e uma intenção com propósito com vista ao alívio desse sofrimento (Dalai Lama, 2001).

Gilbert (e.g., 2005) conceptualiza a compaixão como uma atitude universal nos humanos, produto da evolução do sistema fisiológico subjacente à vinculação e de prestação de cuidados, sendo ainda entendida como uma competência que pode ser promovida através de treino específico. A compaixão define-se, assim, por uma combinação de motivos, pensamentos, emoções e ações de abertura empática, calorosa e nãoavaliativa perante o sofrimento (do próprio ou dos outros) e pela procura ativa para o seu alívio (Gilbert, 2005, 2009). De acordo com o postulado por Gilbert e colaboradores (2017), a compaixão definida como uma sensibilidade e atitude intencional de compromisso para o cuidado e alívio ao sofrimento engloba duas dimensões: ( $l$ ) os atributos e motivações compassivas e (ii) as ações compassivas. As motivações compassivas definem-se como uma competência ou motivação para lidar com o sofrimento numa atitude de sensibilidade e aceitação (Gilbert, 2009). Estas motivações compassivas traduzem-se no cuidado para $O$ bem-estar, isto é, pelo carinho pelo próprio e pelos outros quando estão em dificuldades e pela manifestação de um interesse e conhecimento genuínos pelas origens do sofrimento, estando na base das ações compassivas. Estas ações compassivas dizem respeito à forma como se age de acordo com as motivações compassivas, focando-se deliberadamente a atenção no que pode ser útil, perante experiências indesejáveis, desafiantes ou dolorosas (Gilbert, 2009, 2010).

Nesta conceptualização, são identificados diferentes fluxos ou direções para a compaixão (Gilbert, 2009, 2010; Gilbert, McEwan, Matos, \& Rivis, 2011): direcionada em relação aos outros, expressa em relação ao self, ou recebida por parte dos outros. A compaixão direcionada ao self (i.e., autocompaixão) pode ser compreendida como uma estratégia adaptativa de regulação emocional que ajuda o indivíduo a lidar com sentimentos dolorosos (e.g., situações de fracassos, insuficiências, falhas ou insucessos) sem os evitar, mas ao invés disso, aceitando-os com gentileza, de forma compreensiva e sem julgamento (Gilbert, 2010). Deste modo, o constructo de autocompaixão não se diferencia da definição mais geral de compaixão, e pressupõe que o indivíduo esteja atento, aberto e sensível ao próprio sofrimento. De acordo com $\operatorname{Neff}(2003,2012)$ esta atitude deve ser distinguida de autorresignação, uma vez que a conceptualização de autocompaixão compreende ainda o desejo de aliviar o sofrimento e de recuperar o bem-estar, através da experiência de sentimentos de cuidado, gentileza e de bondade para consigo próprio.

Adicionalmente, a autocompaixão baseia-se no pressuposto de que a imperfeição é parte integrante da condição humana (Neff, 2003). Esta dimensão de humanidade comum parece facilitar a partilha de experiências comuns mesmo quando indesejáveis ou dolorosas, promovendo sentimentos de ligação segura e de conexão ao outro e minimizando sentimentos de isolamento (Neff, 2012). A autocompaixão 
parece funcionar como uma bolha de emotional safeness dentro da qual os indivíduos se percebem a si, assim como às circunstâncias da vida, numa atitude de abertura e sem autocondenação (Leary, 2004). Adicionalmente, a autocompaixão associa-se a níveis mais elevados de bem-estar e satisfação com a vida, promovendo relações interpessoais mais positivas, maior sentido de pertença e ligação ao outro ou social safeness (Akin \& Akin, 2015).

O constructo de social safeness caracteriza-se por uma perceção do mundo social como seguro, apaziguador e calmo, capaz de induzir sentimentos de pertença e uma perceção de aceitação por parte dos outros. Este sentimento de pertença e de conexão social tem sido associado a um bom ajustamento psicológico e bem-estar (Gilbert, 2009, 2010, 2015), assim como a outros indicadores positivos (e.g., pensamento criativo, capacidade de resolução de problemas e comportamentos pró sociais; Rothstein \& Uslaner, 2005). De facto, a necessidade de pertença pode ser compreendida como uma necessidade humana universal crucial para o bem-estar dos indivíduos (Baumeister \& Leary, 1995; Kelly, Zuroff, Leybman, \& Gilbert, 2012).

Existe um consenso alargado de que o estabelecimento de alianças e relações de amizade têm um papel fundamental na saúde física e mental (Cacioppo \& Patrick, 2008), por contraste, o isolamento, o sentimento de alienação, a falta de alianças e de cooperação são altamente prejudiciais ao bem-estar. Investigadores defendem que a experiência de rejeição (seja objetiva ou subjetiva) e de desconexão pode ter um impacto negativo na expressão genética das células imunitárias, gerando uma cascata de acontecimentos neurobiológicos com repercussões a vários níveis (nomeadamente num aumento da sintomatologia depressiva; Beck \& Bredemeier, 2016). De acordo com Gilbert (2010), a solidão pode ativar o sistema de defesa/ameaça e gerar um medo poderoso que serve como um sinal de que é necessário restabelecer laços sociais quebrados e evitar vários tipos de dificuldades ou perigos.

Dados empíricos recentes sugerem que indivíduos que se sentem mais seguros, aceites e ligados aos outros, tendem a apresentar níveis mais baixos de autocriticismo e isolamento social (e.g., Kelly et al., 2012). Por outro lado, os indivíduos que se sentem inseguros na sua envolvente social tendem a fazer uma avaliação negativa do self, o que parece influenciar a sua capacidade de ler sinais sociais acabando por se afastar mais dos outros e perpetuando sentimentos de insegurança e hipervigilância à ameaça (Kelly et al., 2012), maior dificuldade em regular as emoções, as quais parecem explicar níveis mais elevados de sintomatologia depressiva (Cacioppo \& Patrick, 2008; Gilbert, 2010, 2015).

Vasta literatura tem vindo a enfatizar o papel central de diferentes processos de regulação emocional na explicação de diferentes indicadores de psicopatologia, sublinhando o evitamento experiencial como um processo mal-adaptativo central para a explicação da sintomatologia depressiva (e.g., Carvalho, Dinis, Pinto-Gouveia, \& Estanqueiro, 2015; Chawla \& Ostafin, 2007; Spinhoven, Drost, De Rooij, Van Hemert, \& Penninx, 2016).

De acordo com a Terapia da Aceitação e do Compromisso (ACT), o evitamento experiencial é conceptualizado como um processo que envolve a indisponibilidade do indivíduo para estar em contacto com eventos privados aversivos (e.g., pensamentos, emoções, sensações, memórias) e esforços deliberados para alterar a intensidade, frequência e duração ou o contexto que os ocasiona, mesmo que isso afete a vivência de valores pessoais e induza ações incongruentes com esses mesmos valores (Hayes, Strosahl, \& Wilson, 1999; Hayes, Wilson, Gifford, Follette, \& Strosahl, 1996). O evitamento experiencial é considerado uma estratégia mal-adaptativa de lidar com emoções desagradáveis, pois apesar de produzir alívio imediato 
em relação às experiências emocionais intensas, a longo prazo tem um efeito paradoxal (Chapman, Gratz, \& Brown, 2006). De facto, apesar de em certas situações o evitamento experiencial ser uma estratégia adaptativa, por providenciar proteção ao indivíduo, este processo tende a ser mal-adaptativo à medida que o padrão de resposta se torna rígido, consumindo recursos (e.g., tempo, esforço e energia) e comprometendo objetivos de vida valorizados (Kashdan, Barrios, Forsyth, \& Steger, 2006). Paralelamente, o evitamento experiencial tende a funcionar como uma barreira à experienciação de interações sociais afiliativase significativas (Kashdan et al., 2014).

Embora a literatura tenha vindo a enfatizar o impacto das experiências precoces de calor e segurança e autocompaixão na psicopatologia (e.g., depressão), vários fatores e processos de regulação emocional estão envolvidos nestas relações (Gilbert, 2005; Gilbert, Baldwin, Irons, Baccus, \& Palmer, 2006; Schore, 2001). A relação entre experiências emocionais precoces e a autocompaixão está bem documentada. No entanto, a relação entre estas vivências precoces positivas e a tendência para adotar estratégias de evitamento experiencial não está clarificada, embora alguns dados empíricos apontem para a existência de uma relação significativa entre experiências precoces negativas e evitamento experiencial (Batten, Follette, \& Aban, 2002; Gratz, Bornovalova, Delany-Brumsey, Nick, \& Lejuez, 2007). O objetivo desta investigação é explorar o impacto das experiências precoces de calor e afeto e das competências autocompassivas na sintomatologia depressiva, assim como o efeito mediador da proximidade ao outro e do evitamento experiencial nesta relação. Adicionalmente, é examinada a diferença entre homens e mulheres nas relações testadas. É colocada a hipótese de que os indivíduos que reportam mais memórias emocionais positivas em relação às suas vivências precoces com pais/cuidadores e apresentam maiores competências autocompassivas tendem a apresentar menores níveis de sintomatologia depressiva. Adicionalmente é esperado que as relações entre memórias emocionais precoces e competências autocompassivas com a sintomatologia depressiva sejam mediadas por níveis superiores de ligação e proximidade ao outro e menor evitamento experiencial face a eventos internos. Finalmente, é plausível que estas relações sejam comuns tanto para o sexo masculino como para o sexo feminino, e que as variáveis e processos em estudo operem de forma semelhante para a explicação da sintomatologia depressiva em ambos os sexos.

\section{Método}

\section{Participantes}

A amostra deste estudo foi composta por 389 adultos da população geral (124 homens e 265 mulheres), com idades compreendidas entre os 18 e os 50 anos. A idade média apresentada pelos participantes do sexo masculino foi de $31,46(D P=10,97)$ e nas participantes do sexo feminino de $32,02(D P=10,98)$. Em relação à escolaridade, o grupo do sexo masculino apresentou uma média de 12,63 ( $D P=3,34)$ e o grupo feminino de 14,00 ( $D P=3,38)$ anos de escolaridade. No que concerne às diferenças entre os dois grupos, não foram encontradas diferenças estatisticamente significativas na variável idade $\left[t_{(387)}=-0,471 ; p=0,638\right]$, no entanto, observaram-se diferenças estatisticamente significativas quanto aos anos de escolaridade [ $t_{(386)}$ $=-3,789 ; p<0,001]$. 


\section{Medidas}

Escala de Memórias Precoces de Calor e Afeto (Early Memories of Warmth and Safeness Scale [(EMWSS); Richter et al. (2009); Matos, Pinto-Gouveia e Duarte (2014)]. Esta medida de autorresposta, constituída por 21 itens, visa medir as memórias de experiências emocionais de calor, segurança e afeto sentidas/experienciadas na infância. É solicitado aos participantes que respondam aos itens (e.g., "Sentia que era um membro querido da minha família"), utilizando uma escala de cinco pontos (de 0 = "Não, nunca" a 4 = "Sim, a maior parte do tempo"). A EMWSS revelou boas características psicométricas, apresentando uma consistência interna muito boa ( $\alpha$ de Cronbach $=0,97$ ) tanto na versão original (Richter et al., 2009) quanto na versão portuguesa (Matos et al., 2014).

Escala de Atributos e Ações Compassivas [Compassionate Engagement and Actions Scales [(CEAS); Gilbert et al. (2017)]. Esta medida de autorresposta é composta por três escalas que medem a compaixão, perante situações de dificuldade ou sofrimento: ( $)$ Escala de Autocompaixão (CEAS-SC); (ii) Escala de Compaixão pelos Outros, e (iii) Escala de Receber Compaixão dos Outros. Tendo em conta o objetivo específico desta investigação, apenas foi usada a escala de autocompaixão (CEAS-SC) para avaliar a compaixão dirigida ao self. A escala é composta por 13 itens e integra duas seções distintas: 1) "atributos autocompassivos" (com 8 itens, desenhada para avaliar a motivação e capacidade para lidar com as situações difíceis e dolorosas com uma atitude calorosa e de aceitação); 2) "ações autocompassivas" (composta por 5 itens, que avaliam as ações eficazes/úteis, compassivas e tolerantes para lidar com pensamentos e emoções negativas em situações de dificuldade ou sofrimento). A CEAS-SC apresenta uma escala de resposta dez pontos, variando entre 1 ("Nunca") e 10 ("Sempre"), na qual valores mais elevados representam níveis superiores de autocompaixão. O estudo original (Gilbert et al., 2017), o qual apresenta a validação desta medida para a população Americana, Britânica e Portuguesa, revela que a CEAS-SC apresenta boas caraterísticas psicométricas, com alfas de Cronbach que variam entre 0,74 e 0,84.

Escala de Proximidade e Ligação aos Outros [Social Safeness and Pleasure Scale (SSPS); Gilbert et al. (2009); Pinto-Gouveia, Matos e Dinis (2008)]. Esta escala de autorresposta, de 11 itens, avalia o grau em que as pessoas se sentem aceites, seguras e acarinhadas no seu grupo social. É solicitado aos participantes que assinalem para cada item (e.g., "Sinto-me aceite pelas outras pessoas") a frequência com que experienciam sentimentos positivos de pertença, ligação e aceitação na interação social, utilizando uma escala de cinco pontos (de 1 = "Quase nunca" a 5 = "Quase sempre"). A SSPS apresenta uma consistência interna muito boa ( $\alpha$ de Cronbach = 0,91), tanto na versão original (Gilbert et al., 2009) como na versão portuguesa (Pinto Gouveia et al., 2008).

Questionário de Aceitação e Ação //[Acceptance and Action Questionnaire - //(AAQ-II); Bond et al. (2011); Pinto-Gouveia, Gregório, Dinis e Xavier (2012)]. Esta medida de autorresposta constituída por sete itens, visa medir o grau de evitamento experiencial, isto é, a indisponibilidade para estar com as experiências internas consideradas aversivas, perda de contacto com o momento presente e ausência de comportamentos comprometidos com os valores. É solicitado aos participantes que assinalem a sua concordância em relação aos itens (e.g." "Tenho medo dos meus sentimentos"), utilizando uma escala de sete pontos (de 1 = "Nunca verdadeiro" a 7 = "Sempre verdadeiro"). Esta escala mostrou boas características psicométricas, apresentando uma consistência interna muito boa ( $\alpha$ de Cronbach $=0,84$ ), tanto na versão original (Bond et al., 2011), como na versão portuguesa (Pinto Gouveia et al., 2012).

Escala de Ansiedade, Depressão e Stress[Depression Anxiety Stress Scales (DASS-21); Lovibond e Lovibond (1995); Pais-Ribeiro, Honrado e Leal (2004)]. Composta por três subescalas com sete itens cada uma, a 
DASS-21 é a versão curta da DASS-42 (Lovibond \& Lovibond, 1995) e visa avaliar o grau de ansiedade, depressão e stresse experienciados. É solicitado aos participantes que assinalem, utilizando uma escala de quatro pontos (de 0 = "Não se aplicou nada a mim" a 3 = "Aplicou-se a mim a maior parte das vezes"), a frequência com que sentiram emoções negativas durante a última semana. Tendo em conta os objetivos da presente investigação, utilizou-se apenas a subescala depressão (DASS-21-DEP), a qual revelou boas características psicométricas, tanto na versão original ( $\alpha$ de Cronbach = 0,87; Lovibond \& Lovibond, 1995), quanto na versão portuguesa ( $\alpha$ de Cronbach = 0,80; Pais-Ribeiro et al., 2004).

Os alfas de Cronbach respeitantes a estas medidas encontram-se na Tabela 1.

\section{Procedimento}

O presente estudo respeitou todas as normas éticas e os princípios deontológicos inerentes à investigação em psicologia, nomeadamente na recolha da amostra e tratamento dos dados. Após o consentimento da comissão de ética da instituição de acolhimento da presente investigação, a amostra foi recolhida através de uma plataforma online (LimeSurvey).

O convite à participação neste estudo foi partilhado através de redes sociais (Facebook) e através de mensagem privada para contactos pessoais. No convite constava uma breve descrição dos objetivos e procedimentos do estudo, realçando a importância do estudo em questão, o qual se encontra inserido numa investigação mais alargada sobre diferentes processos de regulação emocional e qualidade de vida, bem como os critérios de inclusão do estudo (homens e mulheres com idades superiores a 18 anos). Adicionalmente, foi dado a todos os participantes informação acerca do caráter voluntário da sua participação, da confidencialidade e anonimato das respostas. Os investigadores comprometeram-se ainda a utilizar os dados fornecidos pelos participantes estritamente para fins de investigação, assim como a prestar o esclarecimento sobre quaisquer dúvidas acerca da mesma, em qualquer momento. Este convite incluía ainda um link que direcionava para a versão online do protocolo de investigação constituído por: consentimento informado, questionário de recolha de informação biográfica e as medidas de autorresposta anteriormente descritas.

\section{Estratégia Analítica}

A análise de dados foi elaborada com recurso ao software IBM SPSS Statistics 22.0 do SPSS (SPSS IBM; Chicago, IL) e com recurso ao softwareAMOS (Analysis of Momentary Structure, Version 23.0; Arbuckle, 2014).

De forma a analisar as características da amostra nas variáveis em estudo, nomeadamente, as médias e desvios-padrão, foram conduzidas análises estatísticas descritivas. Com vista a compreender as associações entre os diferentes constructos da investigação, procedeu-se a análises de correlação de Pearson. A leitura e análise das magnitudes dos resultados seguiram os princípios e recomendações de Cohen, Cohen, West e Aiken (2003), segundo as quais se considera de magnitude fraca correlações entre 0,1 e 0,3, moderada entre 0,3 e 0,5 e forte iguais ou superiores a 0,5, considerando-se um nível de significância de 0,05.

Por forma a testar as relações estruturais presumidas entre as variáveis do modelo proposto, foi elaborada uma equação de modelos estruturais (SEM) (MacKinnon, 2008). O modelo testou o efeito indireto das experiências precoces de calor e afeto e das competências autocompassivas (variáveis 
independentes) na sintomatologia depressiva (variável dependente), através da ligação ao outro e do evitamento experiencial (variáveis mediadoras). Foi utilizado o método de Máxima Verosimilhança para testar os coeficientes de regressão e uma série de testes que visam calcular a qualidade do ajustamento do modelo (CFI, TLI, NFIe RMSEA; Hu \& Bentler, 1999). Os efeitos indiretos estabelecidos entre as variáveis foram testados a partir de bootstrapping (com 5000 amostras), com um intervalo de confiança corrigido de $95 \%$ do efeito indireto. Nesta análise, considera-se que o efeito indireto é significativo quando o intervalo de confiança (/C) não inclui o zero (Kline, 2005).

Por fim, foi realizada uma análise de multigrupos de forma a testar a existência de diferenças entre homens e mulheres no modelo final.

\section{Resultados}

\section{Análise preliminar dos dados}

A adequação dos dados foi testada através da análise dos valores de assimetria (Skewness - Sk) e de curtose (Kurtosis - Ku), tendo sido possível confirmar o pressuposto da normalidade da distribuição das variáveis $(|S K|<3$ e $|K u|<8$; Kline, 2005). De facto, os valores de assimetria variaram entre -0,69 (EMWSS) e 0,80 (DASS-21-DEP), e os valores de curtose entre -0,29 (EMWSS) e 1,20 (DASS-21-DEP).

\section{Estatística descritiva e de correlações}

As estatísticas descritivas relativamente às variáveis estudadas, para o total da amostra $(N=389)$, são apresentadas na Tabela 1.

Tabela 1

Alfas de Cronbach ( $\alpha$ ), Médias (M), Desvios-Padrão (DP) e Correlações entre as Variáveis em Estudo

\begin{tabular}{|c|c|c|c|c|c|c|c|}
\hline & $\alpha$ & $M$ & $D P$ & 1 & 2 & 3 & 4 \\
\hline 1. EMWSS & 0,98 & 57,39 & 20,08 & 1 & & & \\
\hline 2. CEAS-SC & 0,88 & 66,68 & 16,05 & $0,31^{* * *}$ & 1 & & \\
\hline 3. SSPS & 0,95 & 41,79 & 8,44 & $0,51^{* * *}$ & $0,46^{* * *}$ & 1 & \\
\hline 4. AAQ II & 0,93 & 22,48 & 9,72 & $-0,32^{* * *}$ & $-0,42^{* * *}$ & $-0,47^{* * *}$ & 1 \\
\hline 5. DASS-21-DEP & 0,94 & 9,29 & 5,62 & $-0,33^{* * *}$ & $-0,32^{* * *}$ & $-0,47^{* * *}$ & $0,52^{* * *}$ \\
\hline
\end{tabular}

Nota EMWSS = Escala de Memórias Precoces de Calor e Segurança; CEAS-SC = Escala de Atributos e Ações Autocompassivas; SSPS = Escala de Proximidade e Ligação ao Outro; AAQ II = Questionário de Aceitação e Ação; DASS-21-DEP = Escala de Ansiedade, Depressão e StressSubescala de Depressão.

${ }^{* * *} p<0,001$.

Os resultados das análises de correlação de Pearson permitiram observar associações significativas entre todas as variáveis em estudo. Particularmente, verificou-se uma associação positiva moderada entre memórias precoces de calor e segurança com os cuidadores (EMWSS) e as motivações e ações autocompassivas (CEAS-SC), as quais se correlacionam positivamente com a ligação e proximidade ao outro (SSPS), com magnitude moderadas a altas, e negativa e moderadamente com o evitamento experiencial (AAQ II) e com a sintomatologia depressiva (DASS-21-DEP). Foi ainda possível verificar que a 
ligação e proximidade ao outro (SSPS) revelou uma relação negativa e moderada com o processo de evitamento experiencial e com a sintomatologia depressiva. Os resultados evidenciaram, igualmente, uma associação positiva com uma magnitude forte entre o processo de evitamento experiencial e a severidade dos sintomas depressivos.

Adicionalmente, foram conduzidas análises de correlação de Pearson por sexo. Os resultados permitiram verificar a inexistência de diferenças, tanto quanto à direção quanto à magnitude das associações entre as variáveis em estudo, nos dois grupos (grupo de participantes do sexo masculino e grupo do sexo feminino). Este resultado validou a utilização da amostra global nas análises subsequentes.

\section{Análises de caminhos (path analysis)}

Com o objetivo de compreender mais aprofundadamente as relações entre as variáveis em estudo foi realizada uma análise de modelos estruturais, a análise de caminhos (Path analysis, MacKinnon, 2008). Com base na revisão da literatura, o modelo testado colocou como hipótese que memórias emocionais precoces de calor e afeto e competências autocompassivas assumem um efeito negativo na explicação da sintomatologia depressiva através de níveis superiores de proximidade e ligação ao outro e de níveis inferiores de evitamento experiencial.

O modelo foi testado através de um modelo saturado que consistia em 20 parâmetros. Este modelo inicial explicou 36\% da variância da ligação e proximidade ao outro (medida através da SSPS), 22\% da variância do evitamento experiencial (AAQ II) e 34\% da sintomatologia depressiva (DASS-21-DEP) (Figura 1).

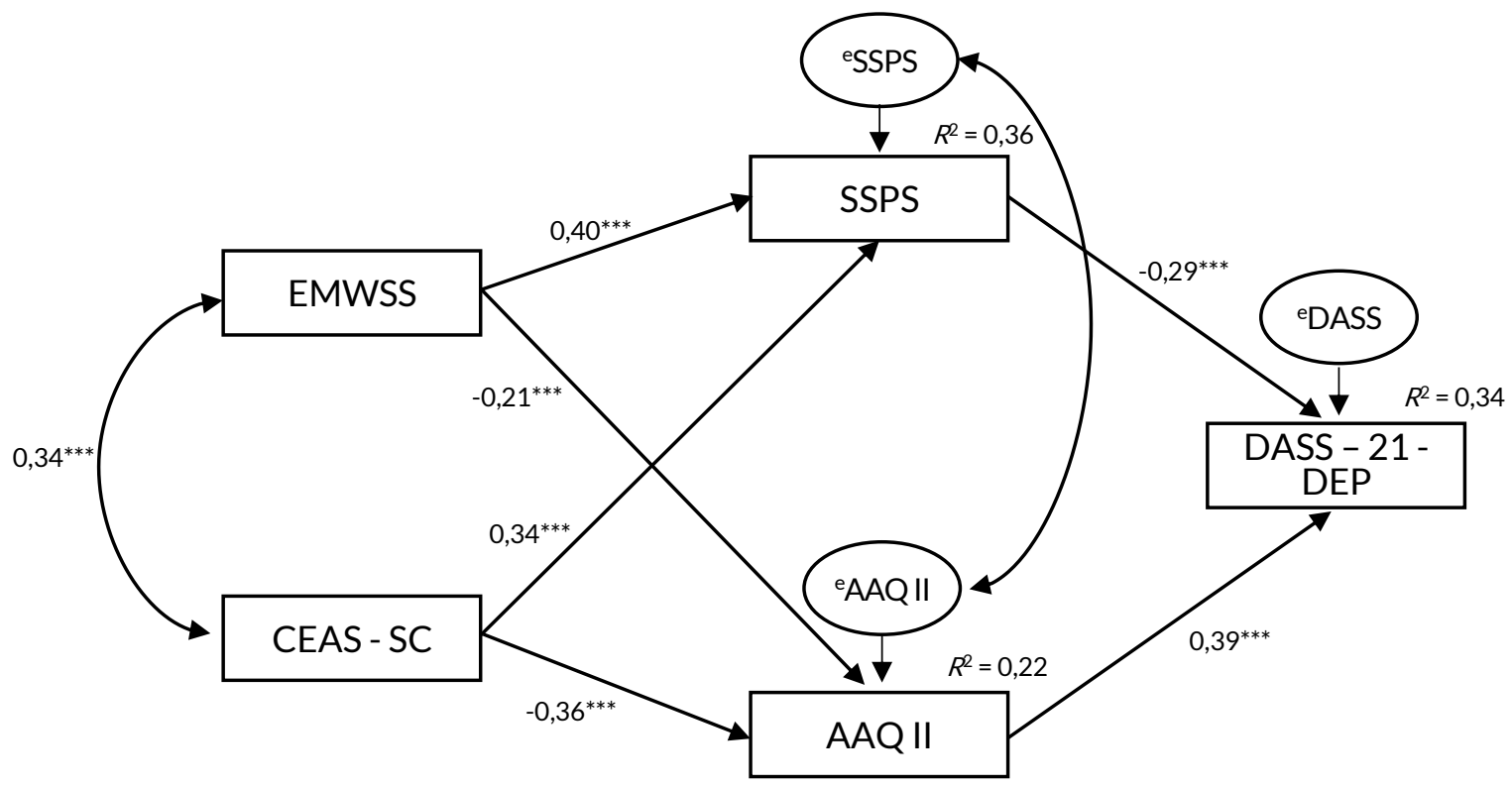

Figura 1. Modelo final. Coeficientes estandardizados dos caminhos (paths) entre as variáveis. Todas os coeficientes dos caminhos revelaram-se significativos para um nível de $0,05\left({ }^{* * *} p<0,001\right)$; EMWSS = Escala de Memórias Precoces de Calor e Segurança; CEAS-SC = Escala de Atributos e Ações Autocompassivas; SSPS = Escala de Proximidade e Ligação ao Outro; AAQ II = Questionário de Aceitação e Ação; DASS-21-DEP = Escala de Ansiedade, Depressão e Stress-Subescala de Depressão. 
A análise dos resultados mostrou que dois dos caminhos (paths) não se revelaram estatisticamente significativos. Especificamente estes caminhos foram: o efeito direto da CEAS-SC na DASS-21-DEP ( $b_{\text {CEAS_SC }}=0,009 ; S E b=0,17 ; Z=-0,554 ; p=0,580 ; \beta=-0,27$ ) e o efeito direto da EMWSS na DASS-21-DEP $\left(b_{\mathrm{EMWSS}}=-0,23 ;\right.$ SEb $\left.=0,013 ; Z=-1,755 ; p=0,079 ; \beta=-0,82\right)$.

De acordo com procedimentos recomendados, procedeu-se à eliminação progressiva dos caminhos referidos, tendo o modelo sido reajustado e recalculado. O modelo reajustado demonstrou explicar $36 \%$ da variância na SSPS e $22 \%$ da variância no AAQ II e 34\% da variância da sintomatologia depressiva, e apresentou excelente adequação aos dados empíricos $\left[\chi^{2}{ }_{(2)}=3,375 ; p=0,185, C M I N / D F=1,687 ; T L I=\right.$ 0,986; $C F I=0,997 ; N F I=0,993 ; R M S E A=0,042 ; p=0,458 ;(95 \% / C=0,000-0,118)]$. Adicionalmente, neste modelo todos os caminhos se revelaram significativos. Os resultados demonstraram assim que: a EMWSS revelou um efeito direto na SSPS de 0,339 e no AAQ II de -0,211 ( $b=0,01 ; S E=0,002 ; p<0,001)$. A EMWSS também mostrou um efeito indireto de $-0,197$ na DASS-21-DEP através dos efeitos do AAQ II e da SSPS (95\% IC = -0,272 a -0,133). Quanto à CEAS-SC, esta variável revelou um efeito direto positivo de 0,339 na SSPS e negativo de -0,358 no AAQ II. Esta variável mostrou ainda um efeito indireto de -0,237 na DASS-21DEP através dos efeitos da SSPS e do AAQ II (95\% IC $=-0,299$ a -0,173). No que diz respeito à SSPS, esta variável revelou efeitos diretos de -0,287 na DASS-21-DEP. O AAQ II, demonstrou efeitos diretos de 0,391 na DASS-21-DEP.

Por fim, e por forma a testar a invariância do modelo por sexo, foi realizada uma análise multigrupos. Os resultados obtidos demonstraram a não existência de diferenças significativas em relação aos pesos fatoriais $\left[\chi^{2}(6)=7,98 ; p=0,247\right]$, revelando assim a invariância do modelo nos dois grupos em comparação (homens e mulheres).

Em síntese, os resultados da análise de caminhos sugerem que as memórias precoces de calor e segurança e a existência de competências autocompassivas se associam com níveis mais baixos de sintomatologia depressiva, através de mais sentimentos de proximidade e ligação aos outros e de menor evitamento experiencial, tanto para homens como mulheres.

\section{Discussão}

As experiências emocionais precoces positivas com figuras de vinculação, assim como as motivações e ações autocompassivas têm sido associadas a diferentes indicadores de bem-estar e saúde mental (e.g., DeHart et al., 2006; Gilbert et al., 2017; Irons et al., 2006; MacBeth \& Gumley, 2012; Marta-Simões et al., 2018; Mikulincer \& Shaver, 2005). No entanto, não estão ainda inteiramente clarificados quais os mecanismos mediadores nestas associações. Assim, o presente estudo pretendeu testar o efeito mediador da proximidade e ligação ao outro e do evitamento experiencial na relação entre experiências afiliativas precoces de calor e segurança e as competências autocompassivas na sintomatologia depressiva, numa amostra composta por homens e mulheres da população geral.

Os resultados obtidos estão de acordo com estudos prévios e parecem confirmar as hipóteses previamente apontadas, evidenciando uma relação positiva significativa entre experiências precoces positivas, competências autocompassivas e com a proximidade e ligação ao outro, e negativa com o evitamento experiencial e a sintomatologia depressiva (e.g., Carvalho et al., 2015; Chawla \& Ostafin, 2007; Gilbert et al., 2017; Hayes et al., 1996; Kelly \& Dupasquier, 2016). Este estudo acrescenta à literatura existente a 
evidência da associação positiva moderada entre experiências precoces de calor e afeto e competências autocompassivas, e da relação significativa entre estas competências e menores índices de evitamento experiencial. Embora este seja o primeiro estudo que explora a relação entre competências autocompassivas e evitamento experiencial, os resultados obtidos parecem suportar a abordagem de Yadavaia, Hayes e Vilardaga (2014), que sugere existir paralelismo entre os constructos de flexibilidade psicológica e autocompaixão.

Os resultados da análise de caminhos (path analysis) mostraram que o modelo testado apresenta uma excelente adequação aos dados, explicando $34 \%$ da variância da sintomatologia depressiva. Adicionalmente, os dados obtidos na análise multigrupos suportam a invariância do modelo testado entre homens e mulheres. Particularmente, os dados apontam que as experiências emocionais precoces de calor e afeto e as competências autocompassivas se associam a menor sintomatologia depressiva através de maiores níveis de ligação e proximidade nas relações sociais e de menor evitamento experiencial. Mais especificamente, os resultados parecem indicar que a presença de memórias precoces positivas e competências autocompassivas, perante insuficiências ou falhas do self, se associam a uma maior proximidade ao outro e a um menor envolvimento em estratégias de evitamento experiencial, mecanismos estes que parecem explicam a sintomatologia depressiva. Estes dados apoiam estudos anteriores, sugerindo que as memórias de calor e afeto nas relações precoces com figuras de vinculação se associam positivamente com a proximidade e ligação ao outro e maior tranquilização nas interações futuras (Gilbert, 2005; Kelly \& Dupasquier, 2016). Paralelamente, foi verificada que uma maior proximidade ao outro está associada a níveis mais baixos de sintomatologia depressiva, dado que vai ao encontro de vasta literatura que destaca o papel pernicioso do isolamento social na depressão (Gilbert, 2010; Kelly et al., 2012). Em consonância com estudos prévios (e.g., Carvalho et al., 2015; Chawla \& Ostafin, 2007; Hayes et al., 1996; Kashdan et al., 2006; Spinhoven et al., 2016), o evitamento experiencial associa-se a níveis mais elevados de sintomatologia depressiva. Este dado parece corroborar o efeito paradoxal do evitamento experiencial como uma estratégia mal-adaptativa de lidar com a experiência interna aversiva (Chawla \& Ostafin, 2007; Hayes et al., 1996). Embora teoricamente expectável, é importante sublinhar que os resultados do presente estudo corroboram que estas relações são invariantes em função do sexo, isto é, mostram que estas relações são semelhantes tanto para homens como para mulheres.

O presente estudo apresenta, contudo, algumas limitações. Uma das principais limitações diz respeito ao facto de este ser um estudo transversal, o que impossibilita o estabelecimento de relações causais entre os constructos. Outra das limitações prende-se com o facto de terem sido utilizados exclusivamente instrumentos de autorresposta e por via online, o que se poderá traduzir numa maior subjetividade e consequente enviesamento dos resultados. Estudos futuros deverão explorar a relação entre estas variáveis através de design experimental ou longitudinal, assim como o recurso a entrevistas, de modo a ultrapassar estas limitações. Uma limitação importante a salientar diz respeito ao facto do modelo testado ser limitado, tendo em conta que a sintomatologia depressiva tem uma natureza complexa e multideterminada. De facto, outras variáveis e processos de regulação emocional (como vergonha, autocriticismo, ou fusão cognitiva) poderão estar envolvidos nas relações em análise, contudo, a intenção foi desenhar um modelo parcimonioso que explorasse o papel mediador do evitamento experiencial e da proximidade ao outro na sintomatologia depressiva. 
Os resultados da presente investigação parecem, no entanto, contribuir de forma significativa para a investigação e prática clínica. De facto, embora a literatura tenha evidenciado a importância do treino de uma mente compassiva na redução da sintomatologia depressiva (Gilbert, 2001, 2007, 2010), os mecanismos de mudança ainda são mal compreendidos. Os dados obtidos permitiram corroborar a importância dos processos de afiliação e conexão ao outro e de aceitação das experiências internas enquanto processos mediadores relevantes para a explicação do bem-estar e da saúde mental. Em termos da prática clínica, os dados reforçam a pertinência do desenvolvimento de intervenções baseadas no treino da autocompaixão com vista à redução da sintomatologia depressiva, sugerindo que estes processos de afiliação ao outro e aceitação devem ser foco das intervenções, quer de foro de prevenção para a população geral quer a nível de intervenção para populações clínicas.

Conflito de interesses | Conflict of interest: nenhum | none.

Fontes de financiamento | Funding sources: nenhuma | none.

Contributos | Contributions: MJV: Revisão da literatura; Recolha, inserção e tratamento dos dados; redação do manuscrito. CF: Contributo na redação do manuscrito; Tratamento e discussão dos dados; Revisão da redação final do manuscrito.

Declaração | Acknowledgments: Partes deste trabalho foram apresentadas em cumprimento parcial dos requisitos para o grau de mestre na Faculdade de Psicologia e Ciências da Educação da Universidade de Coimbra, Portugal | Portions of this work have been submitted in partial fulfillment of the requirements for a master degree, at Faculty of Psychology and Education Sciences, University of Coimbra, Portugal.

\section{Referências}

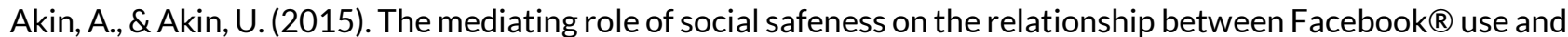
life satisfaction. Psychological Reports, 1172), 341-353. https://doi.org/10.2466/18.07.pr0.117c20z9

Arbuckle, J. L. (2014). Amos (Version 23.0) [Computer Program]. Chicago: IBM SPSS.

Batten, S. V., Follette, V. M., \& Aban, I. B. (2002). Experiential avoidance and high-risk sexual behavior in survivors of child sexual abuse. Journal of Child Sexual Abuse, 102), 101-120. https://doi.org/10.1300/J070v10n02_06

Baumeister, R. F., \& Leary, M. R. (1995). The need to belong: Desire for interpersonal attachments as a fundamental human motivation. Psychological Bulletin, 1173), 497-529. https://doi.org/10.1037/00332909.117.3.497

Beck, A. T., \& Bredemeier, K. (2016). A unified model of depression: Integrating clinical, cognitive, biological, and evolutionary perspectives. Clinical Psychological Science, 4(4), 596-619. https://doi.org/10.1177/2167702616628523

Bond, F. W., Hayes, S. C., Baer, R. A., Carpenter, K. M., Guenole, N., Orcutt, H. K., ... \& Zettle, R. D. (2011). Preliminary psychometric properties of the Acceptance and Action Questionnaire-II: A revised measure of psychological flexibility and experiential avoidance. Behavior Therapy, 42(4), 676-688. https://doi.org/10.1016/j.beth.2011.03.007

Cacioppo, J. T., \& Patrick, W. (2008). Loneliness: Human nature and the need for social connection. New York, NY: W.W. Norton \& Co.

Carvalho, S., Dinis, A., Pinto-Gouveia, J., \& Estanqueiro, C. (2015). Memories of shame experiences with others and depression symptoms: The mediating role of experiential avoidance. Clinical Psychology \& Psychotherapy, 22(1), 32-44. https://doi.org/10.1002/cpp.1862 
Chapman, A. L., Gratz, K. L., \& Brown, M. Z. (2006). Solving the puzzle of deliberate self-harm: The experiential avoidance model. Behaviour Research and Therapy, 44(3), 371-394. https://doi.org/10.1016/j.brat.2005.03.005

Chawla, N., \& Ostafin, B. (2007). Experiential avoidance as a functional dimensional approach to psychopathology: An empirical review. Journal of Clinical Psychology, 63(9), 871-890. https://doi.org/10.1002/jclp.20400

Cohen, J., Cohen, P., West, S. G., \& Aiken, L. S. (2003). Applied multiple regression/correlation analysis for the behavioral sciences ( $3^{\text {rd }}$ ed.). Mahwah, $\mathrm{NJ}$ : Erlbaum.

Dalai Lama. (2001). The compassionate life. Boston: Wisdom Publications.

DeHart, T., Pelham, B. W., \& Tennen, H. (2006). What lies beneath: Parenting style and implicit self-esteem. Journal of Experimental Social Psychology, 42(1), 1-17. https://doi.org/10.1016/j.jesp.2004.12.005

Depue, R. A., \& Morrone-Strupinsky, J. V. (2005). A neurobehavioral model of affiliative bonding: Implications for conceptualizing a human trait of affiliation. Behavioral and Brain Sciences, 28(3), 313-395. https://doi.org/10.1017/s0140525x05000063

Gilbert, P. (2001). Evolutionary approaches to psychopathology: The role of natural defences. Australian and New Zealand Journal of Psychiatry, 35(1), 17-27. https://doi.org/10.1046/j.1440-1614.2001.00856.x

Gilbert, P. (2005). Compassion and cruelty: A biopsychosocial approach. In P. Gilbert (Ed.), Compassion: Conceptualization, research and use in psychotherapy (pp. 9-74). London: Routledge.

Gilbert, P. (2007). The evolution of shame as a marker for relationship security. In J. Tracy, R. Robins, \& J. Tangney (Eds.), The self-conscious emotions: Theory and research (pp. 283-309). New York, NY: Guilford Press.

Gilbert, P. (2009). Introducing compassion-focused therapy. Advances in Psychiatric Treatment, 15(3), 199-208. https://doi.org/10.1192/apt.bp.107.005264

Gilbert, P. (2010). The compassionate mind: A new approach to life's challenges. Oakland: New Harbinger Publications.

Gilbert, P. (2015). The evolution and social dynamics of compassion. Social and Personality Psychology Compass, 96), 239-254. https://doi.org/10.1111/spc3.12176

Gilbert, P., Baldwin, M. W., Irons, C., Baccus, J. R., \& Palmer, M. (2006). Self-criticism and self-warmth: An imagery study exploring their relation to depression. Journal of Cognitive Psychotherapy, 20(2), 183-200. https://doi.org/10.1891/088983906780639817

Gilbert, P., Catarino F., Duarte C., Matos M., Kolts R., Stubbs J., ... Basran, J. (2017). The development of compassionate engagement and action scales for self and others. Journal of Compassionate Health Care, 4(1), 1-24. https://doi.org/10.1186/s40639-017-0033-3

Gilbert, P., Cheung, M. S-P., Grandfield, T., Campey, F., \& Irons, C. (2003). Recall of threat and submissiveness in childhood: Development of a new scale and its relationship with depression, social comparison and shame. Clinical Psychology \& Psychotherapy, 1022), 108-115. https://doi.org/10.1002/cpp.359

Gilbert, P., McEwan, K., Matos, M., \& Rivis, A. (2011). Fears of compassion: Development of three self-report measures. Psychology and Psychotherapy: Theory, Research and Practice, 84(3), 239-255. https://doi.org/10.1348/147608310X526511

Gilbert, P., McEwan, K., Mitra, R., Richter, A., Franks, L., Mills, A., ... Gale, C. (2009). An exploration of different types of positive affect in students and in patients with bipolar disorder. Clinical Neuropsychiatry: Journal of Treatment Evaluation, 6(4), 135-143. Retrieved from http://ccare.stanford.edu/wpcontent/uploads/2014/02/Gilbert-et-al-2009-exploration-of-different-types-positive-affect-in-studentsand-patients1.pdf

Gratz, K. L., Bornovalova, M. A., Delany-Brumsey, A., Nick, B., \& Lejuez, C. W. (2007). A laboratory-based study of the relationship between childhood abuse and experiential avoidance among inner-city substance users: The role of emotional nonacceptance. Behavior Therapy, 38(3), 256-268. https://doi.org/10.1016/j.beth.2006.08.006 
Hayes, S. C., Strosahl, K. D., \& Wilson, K. G. (1999). Acceptance and commitment therapy: An experiential approach to behavior change. New York, NY: Guilford Press.

Hayes, S. C., Wilson, K. G., Gifford, E. V., Follette, V. M., \& Strosahl, K. (1996). Experiential avoidance and behavioral disorders: A functional dimensional approach to diagnosis and treatment. Journal of Consulting and Clinical Psychology, 64(6), 1152-1168. https://doi.org/10.1037/0022-006x.64.6.1152

$\mathrm{Hu}, \mathrm{L.}, \&$ Bentler, P. (1999). Cut-off criteria for fit indexes in covariance structure analysis: Conventional criteria versus new alternatives. Structural Equation Modeling: A Multidisciplinary Journal, 6(1), 1-55. https://doi.org/10.1080/10705519909540118

Irons, C., Gilbert, P., Baldwin, M. W., Baccus, J. R., \& Palmer, M. (2006). Parental recall, attachment relating and self-attacking/self-reassurance: Their relationship with depression. British Journal of Clinical Psychology, 45(3), 297-308. https://doi.org/10.1348/014466505X68230

Kashdan, T. B., Barrios, V., Forsyth, J. P., \& Steger, M. F. (2006). Experiential avoidance as a generalized psychological vulnerability: Comparisons with coping and emotion regulation strategies. Behaviour Research and Therapy, 44(9), 1301-1320. https://doi.org/10.1016/j.brat.2005.10.003

Kashdan, T. B., Goodman, F. R., Machell, K. A., Kleiman, E. M., Monfort, S. S., Ciarrochi, J., \& Nezlek, J. B. (2014). A contextual approach to experiential avoidance and social anxiety: Evidence from an experimental interaction and daily interactions of people with social anxiety disorder. Emotion, 14(4), 769-781. https://doi.org/10.1037/a0035935

Kelly, A. C., \& Dupasquier, J. (2016). Social safeness mediates the relationship between recalled parental warmth and the capacity for self-compassion and receiving compassion. Personality and Individual Differences, 89, 157-161. https://doi.org/10.1016/j.paid.2015.10.017

Kelly, A. C., Zuroff, D. C., Leybman, M. J., \& Gilbert, P. (2012). Social safeness, received social support, and maladjustment: Testing a tripartite model of affect regulation. Cognitive Therapy and Research, 36(6), 815826. https://doi.org/10.1007/s10608-011-9432-5

Kline, R. B. (2005). Principles and practice of structural equation modeling ( $2^{\text {nd }}$ ed.). New York, NY: Guilford Press.

Leary, M. R. (2004). Bringing out the best in the self. In M. Leary (Ed.), The curse of the self: Self-awareness, egotism, and the quality of human life (pp. 185-197). Oxford: Oxford University Press.

Lovibond, S. H., \& Lovibond, P. F. (1995). Manual for the Depression Anxiety Stress Scales (2 ${ }^{\text {nd }}$ ed.). Sydney: Psychology Foundation.

MacBeth, A., \& Gumley, A. (2012). Exploring compassion: A meta-analysis of the association between selfcompassion and psychopathology. Clinical Psychology Review, 32(6), 545-552. https://doi.org/10.1016/j.cpr.2012.06.003

MacKinnon, D. P. (2008). Introduction to statistical mediation analysis. Mahwah, NJ: Erlbaum.

Marta-Simões, J., Ferreira, C., \& Mendes, A. L. (2018). Self-compassion: An adaptive link between early memories and women's quality of life. Journal of Health Psychology, 23(7), 929-938. https://doi.org/10.1177/1359105316656771

Matos, M., Pinto-Gouveia, J., \& Duarte, C. (2014). Psychometric properties of the Portuguese version of the Early Memories of Warmth and Safeness Scale. Manuscript submitted for publication.

Mikulincer, M., \& Shaver, P. R. (2005). Attachment security, compassion, and altruism. Current Directions in Psychological Science, 14(1), 34-38. https://doi.org/10.1111/j.0963-7214.2005.00330.x

Neff, K. D. (2003). Self-compassion: An alternative conceptualization of a healthy attitude toward oneself. Self and Identity, 2(2), 85-101. https://doi.org/10.1080/15298860309032

Neff, K. D. (2012). The science of self-compassion. In C. Germer \& R. Siegel (Eds.), Compassion and wisdom in psychotherapy (pp. 79-92). New York, NY: Guilford Press. 
Pais-Ribeiro, J. L., Honrado, A., \& Leal, I. (2004). Contribuição para o estudo da adaptação portuguesa das escalas de ansiedade, depressão e stress (EADS) de 21 itens de Lovibond e Lovibond [Contribution to the adaptation study of the Portuguese adaptation of the Lovibond and Lovibond Depression Anxiety Stress Scales (EADS) with 21 items]. Psicologia, Saúde \& Doenças, 5(2), 229-239. Retrieved from http://hdl.handle.net/10400.12/1058

Pinto-Gouveia, J., Gregório, S., Dinis, A., \& Xavier, A. (2012). Experiential avoidance in clinical and non-clinical samples: AAQ-II Portuguese version. International Journal of Psychology \& Psychological Therapy, 12(2), 139-156. Retrieved from http://www.ijpsy.com/volumen12/num2/323/experiential-avoidance-in-clinicaland-non-EN.pdf

Pinto-Gouveia, J., Matos, M., \& Dinis, A. (2008). Portuguese version of the Social Safeness and Pleasure Scale. Unpublished manuscript, Faculdade de Psicologia e Ciências da Educação, Universidade de Coimbra, Portugal.

Richter, A., Gilbert, P., \& McEwan, K. (2009). Development of an early memories of warmth and safeness scale and its relationship to psychopathology. Psychology and Psychotherapy: Theory, Research and Practice, 82(2), 171-184. https://doi.org/10.1348/147608308×395213

Rothstein, B., \& Uslaner, E. M. (2005). All for all: Equality, corruption, and social trust. World Politics, 58(1), 4172. https://doi.org/10.1353/wp.2006.0022

Schore, A. N. (2001). Effects of a secure attachment relationship on right brain development, affect regulation, and infant mental health. Infant Mental Health Journal, 22(1-2), 7-66. https://doi.org/10.1002/10970355(200101/04)22:1<7::aid-imhj2>3.0.co;2-n

Spinhoven, P., Drost, J., De Rooij, M., Van Hemert, A. M., \& Penninx, B. W. (2016). Is experiential avoidance a mediating, moderating, independent, overlapping, or proxy risk factor in the onset, relapse and maintenance of depressive disorders? Cognitive Therapy and Research, 402), 150-163. https://doi.org/10.1007/s10608015-9747-8

Yadavaia, J. E., Hayes, S. C., \& Vilardaga, R. (2014). Using acceptance and commitment therapy to increase selfcompassion: A randomized controlled trial. Journal of Contextual Behavioral Science, 3(4), 248-257. https://doi.org/10.1016/j.jcbs.2014.09.002 